\title{
Neural Correlates of Successful Encoding Identified Using Functional Magnetic Resonance Imaging
}

\author{
Paul J. Reber, ${ }^{1,3}$ Robert M. Siwiec, ${ }^{1}$ Darren R. Gitleman, ${ }^{2,3}$ Todd B. Parrish, ${ }^{2,3}$ M.-Marsel Mesulam, ${ }^{3}$ and \\ Ken A. Paller ${ }^{1,3}$ \\ Departments of ${ }^{1}$ Psychology and ${ }^{2}$ Radiology and the ${ }^{3}$ Cognitive Neurology and Alzheimer's Disease Center, \\ Northwestern University, Evanston, Illinois 60201
}

Neural activity that occurs during the creation of a new memory trace can be observed using functional magnetic resonance imaging (fMRI). Event-related designs have been used to demonstrate that activity in prefrontal and medial temporal lobe areas is associated with successful memory storage. Here we contrasted activity associated with encoding success and encoding effort. Participants viewed a series of 150 words but attempted to remember only half of them. Encoding effort was manipulated using a cue in the form of a letter ( $R$ or $F$ ) presented after each word to instruct participants either to remember or to forget that word. Increased activity in left inferior prefrontal cortex was observed when words were followed by the cue to remember. In contrast, increased left medial temporal lobe activity was observed for words that were successfully recalled later. These results show that $\mathrm{fMRI}$ correlates of the intention to encode a word are different from fMRI correlates of whether that encoding is successful. Prefrontal activation was strongly associated with intentional verbal encoding, whereas left medial temporal activation was crucial for the encoding that actually led to successful memory on the subsequent test.

Key words: episodic memory; medial temporal lobe; prefrontal cortex; event-related fMRl; subsequent memory; encoding effort
Neuropsychological evidence indicates that memory for recently experienced episodes depends critically on a number of interconnected brain regions, including the medial temporal lobe (MTL) and prefrontal cortex (Squire and Knowlton, 2000; Squire and Schacter, 2002). Although lesion evidence has been useful for implicating brain regions that are necessary for the expression of episodic memories, it is more difficult to ascertain whether these regions make their contributions to the formation, storage, and retrieval of memories. Functional imaging allows examination of the contributions of various brain regions to memory and of how coordination between multiple processes supports memory performance. In the present study, event-related functional magnetic resonance imaging (fMRI) was used to investigate the neural correlates of memory encoding.

Neural events responsible for the formation of episodic memories have been investigated previously (for review, see Paller and Wagner, 2002). Wagner et al. (1998) acquired fMRI data while participants made concrete/abstract judgments about words. Words subsequently recognized with high confidence, compared with forgotten words, were associated with encoding-related activity in left inferior prefrontal cortex (LIPFC) (BA 44, 45, and 47) and left parahippocampal gyrus (BA 35/36). Brewer et al. (1998) devised a similar experiment in which participants performed indoor/outdoor discriminations on visual scenes. Subsequent recognition was associated with increased activation of right prefrontal cortex and bilateral parahippocampal cortex. In both cases, increased MTL activity predicted encoding success,

Received Feb. 15, 2002; revised July 31, 2002; accepted Aug. 21, 2002.

This research was supported by National Institutes of Health Grants R01MH58748 (P.J.R.) and R01-NS34639 (K.A.P.). We thank Patrick Skosnik and Sara Polis for assistance with data collection, data analysis, and manuscript preparation.

Correspondence should be addressed to Dr. Paul J. Reber, Department of Psychology, Northwestern University, 2029 Sheridan Road, Evanston, IL 60201. E-mail: preber@northwestern.edu.

Copyright $@ 2002$ Society for Neuroscience $0270-6474 / 02 / 229541-08 \$ 15.00 / 0$ whereas the laterality of this effect was stimulus specific (left hemisphere for words, right hemisphere for pictures). Encoding processes varied across items because of fluctuations in participants' attention to the stimuli, attention to the task, or the use of particular processing strategies such as semantic elaboration. Furthermore, certain stimuli may precipitate successful memory encoding attributable to distinctiveness or idiosyncratically salient features. Therefore, it is advantageous to combine the subsequent memory methodology, as used in these previous experiments, with experimental manipulations that specifically influence encoding.

In the present study, we monitored neural activity associated with encoding and analyzed the influence of two factors. The first was whether later recognition was successful. The second was the extent to which the participant intended to memorize the stimulus information. Intention to remember was manipulated with a procedure known as directed forgetting (DF). Typical DF procedures entail cues to participants to remember some items and forget others. DF instructions at encoding can have a robust influence on subsequent memorability (Bjork, 1989; Golding and MacLeod, 1998).

Participants viewed words and faces and were instructed to remember or forget individual items on the basis of a cue presented $1 \mathrm{sec}$ after each stimulus. The $1 \mathrm{sec}$ delay gave participants adequate time to perceive, identify, and attend to each study item before knowing whether to remember or forget. For analysis, trials were sorted on the basis of the instruction (remember/ forget) as well as on the subsequent memory outcome, assessed with a post-scan recognition test. We thus contrasted encoding intention and encoding success.

\section{MATERIALS AND METHODS}

Participants. Twelve right-handed volunteers ( 9 women, 3 men) between the ages of 18 and 25 years $($ mean $=20$ ) were recruited from the 

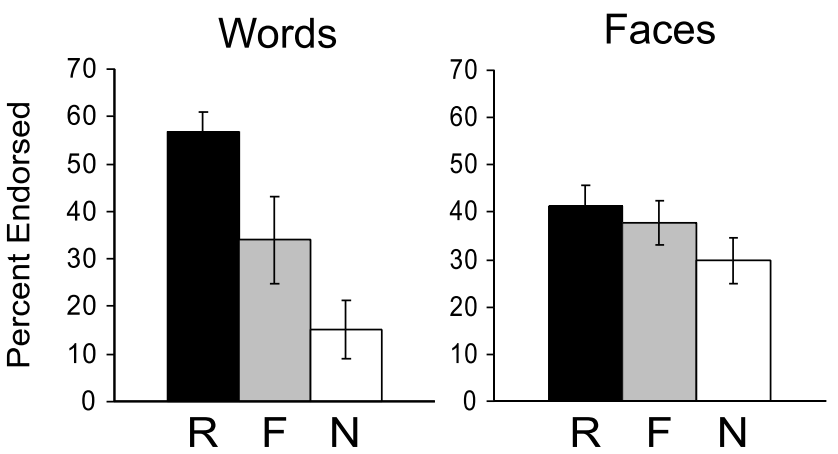

Figure 1. Recognition performance for word and face stimuli that had been studied while fMRI data were collected. Three types of items were included on the recognition test: stimuli that had been cued to be remembered $(R)$, stimuli that had been cued to be forgotten $(F)$, and novel stimuli $(N)$. Endorsing the items as old is the correct response for the $\mathrm{R}$ and $\mathrm{F}$ stimuli and reflects the false alarm rate for the $\mathrm{N}$ stimuli. Error bars indicate the SEM.

Northwestern University community and screened for compatibility with MRI scanning. Data from six other participants were eliminated after testing because of errors in stimulus presentation. All participants were native speakers of English. Each participant was in good health and free from neurological and psychiatric problems. All participants gave informed consent, and the study was approved by the Institutional Review Board of Northwestern University.

Stimulus materials. Word stimuli were selected from the MRC Psycholinguistic Database (http://www.psy.uwa.edu.au/MRCDataBase/uwa mrc.htm). Words ranged in frequency from 30 to 40 occurrences per million (Kucera and Francis, 1967) and in length from 5 to 10 characters. One hundred fifty words were chosen at random for the study phase. Face stimuli were scanned from a 1997 high school yearbook (R. M. Siwiec), and 150 were chosen at random for the study phase. An additional 150 words and 150 faces not used in the study phase were used as foils in the memory test.

Procedure. On each trial, a word or a face was shown for $1000 \mathrm{msec}$, followed by either a cue to remember (a green " $R$ ") or a cue to forget (a red "F") for $1500 \mathrm{msec}$ and then by a $500 \mathrm{msec}$ intertrial interval during which a fixation cross was shown. Participants were instructed to watch all stimuli but to remember only stimuli followed by the remember cue. To ensure that a minimum level of attention was paid to each stimulus, we required participants to respond on each trial by indicating with a button press whether they had seen a face or word. A short demonstration was used to familiarize participants with the stimuli and cues. They were then situated in the MR scanner, as described below, and the study phase was administered in five scanning runs. Each run included 30 words and 30 faces, half with a remember cue and half with a forget cue. To enable the separation of individual hemodynamic responses by deconvolution, an additional 30 trials in each run consisted of only the fixation stimulus for $3 \mathrm{sec}$. Randomized trial orders were selected to maximize the separation of both cue and stimulus effects.

A recognition memory test was administered $\sim 20$ min after the completion of the study task, outside of the scanner. Participants were told that the test contained words and faces that were either novel or from the study phase ("old"). For each test item shown on a computer screen, participants were instructed to use two keys to indicate whether the stimulus was novel or old. Participants were instructed to make these responses regardless of whether the stimulus had been followed by a remember or forget cue and to respond as quickly and accurately as possible.

Imaging methods. A Siemens Vision 1.5-T magnet and head coil were used. The subject's head was comfortably secured using padding and a vacuum-immobilizer device. Stimuli were projected onto a rearprojection screen and viewed through a mirror. Whole brain $\mathrm{T} 2{ }^{*}$ weighted gradient-recalled echoplanar images were collected during the study task $[246 \mathrm{~mm}$ slices; repetition time $(\mathrm{TR})=2000 \mathrm{msec}$; echo time $(\mathrm{TE})=40 \mathrm{msec}$; flip angle $=85^{\circ}$; field of view $\left.(\mathrm{FOV})=24 \mathrm{~cm}\right]$. Slices were oriented along the line connecting the anterior and posterior commissure (AC/PC line; slightly oblique from transverse) with a resolution of $3.75 \times 3.75 \times 6 \mathrm{~mm}$. In each run, 154 whole-brain volumes were collected (4 initial volumes to reach steady state, 135 volumes during the study phase, and an additional 15 volumes at the end of the scan to collect the residual hemodynamic responses of the final trials). For anatomical localization, 3DFLASH $\mathrm{T}_{1}$-weighted images $(\mathrm{TR}=15 \mathrm{msec}$; $\mathrm{TE}=5.6$ msec; flip angle $=20^{\circ}, 1601 \mathrm{~mm}$ axial slices; FOV $=240 \mathrm{~mm} ; 256 \times 256$ matrix) were acquired after the study phase.

Image analysis. Images were coregistered through time using a threedimensional registration algorithm (Cox, 1996). Functional volumes were spatially smoothed with a $7.5 \mathrm{~mm}$ full-width half-maximum Gaussian kernel to improve the signal-to-noise and accommodate residual anatomical differences across participants. Within each run, voxels were eliminated if signal magnitude changed $>10 \%$ between time points $(\mathrm{TR}=2$ sec) or if the mean signal level was below a threshold. Each of the runs was then transformed (Collins et al., 1994) to conform approximately to the atlas of Talairach and Tournoux (1988) (using the MNI-305 reference model) with a final resolution of $2.5 \mathrm{~mm}^{3}$. The five runs of functional data were concatenated into a single time series for each participant. The average response to each trial type was estimated using a general linear model analysis (D. Ward, "Deconvolution Analysis of fMRI Time Series Data," http://afni.nimh.nih.gov/afni) that included the onset of each trial type and several control variables (average signal and linear drift estimated individually for each of the five runs, and estimates
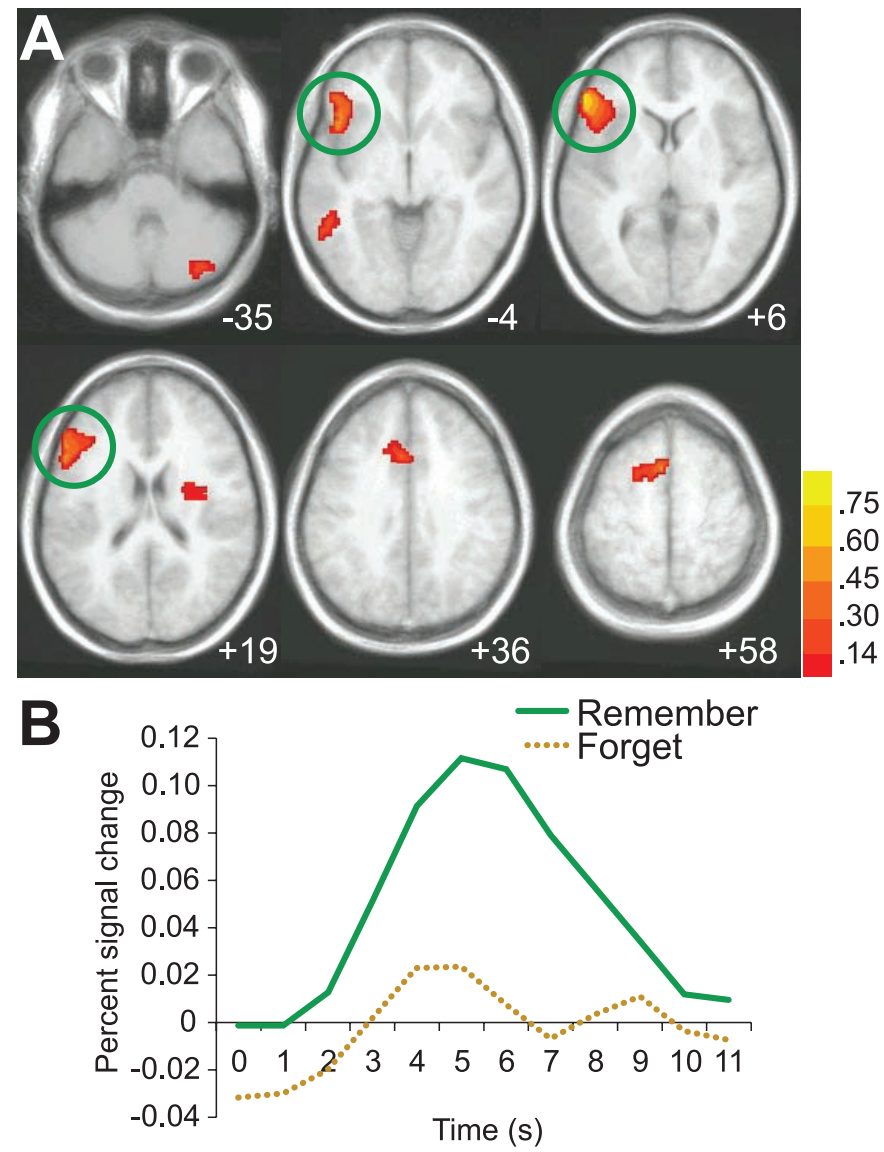

Figure 2. A, Brain areas in which increased activity was observed during word stimuli cued to be remembered (R-words) compared with word stimuli cued to be forgotten (F-words). Regions showing a reliable increase in activity across the group are shown in color overlaid on axial slices from the averaged high-resolution structural images. Regions shown are those for which the difference in peak activity between $\mathrm{R}$ words and $\mathrm{F}$ words was consistently greater than zero across the group of participants $\left(t_{(11)}>3.5\right.$ in a cluster $>500 \mathrm{~mm}^{3}$ in volume). Color intensity (red/orange/yellow) indicates the magnitude of the average signal change between the two conditions. The level of the images within the standard atlas is noted. $B$, Time course of observed activity in the left inferior prefrontal cortical region indicated within the green circle on $A$ for remember and forget trials. The time course shown reflects averaged estimates of activity for each time point across all voxels in the region for all participants (with slight temporal smoothing for display purposes). 
Table 1. Regions that exhibited greater activity for remember trials than forget trials for word stimuli

\begin{tabular}{|c|c|c|c|c|c|}
\hline Brain region & $\begin{array}{l}\text { Brodmann's } \\
\text { area (BA) }\end{array}$ & $x$ & $y$ & $z$ & $\begin{array}{l}\text { Volume } \\
\left(\mathrm{mm}^{3}\right)\end{array}$ \\
\hline $\begin{array}{l}\text { Left inferior prefrontal cortex } \\
\text { Includes areas from }-51,18,22 \text { (BA 9) } \\
\quad \text { to }-44,18,-7 \text { (BA } 47)\end{array}$ & BA $9,45,46,47$ & -48 & 26 & 9 & 7188 \\
\hline Anterior cingulate & BA 32 & -6 & 21 & 33 & 1047 \\
\hline Medial superior frontal gyrus & BA 6 & -9 & 9 & 59 & 1016 \\
\hline Left middle temporal gyrus & BA 37 & -52 & -46 & -6 & 641 \\
\hline Right cerebellum & & 33 & -76 & -35 & 625 \\
\hline Right insula & BA 13 & 31 & -5 & 17 & 578 \\
\hline
\end{tabular}

of corrected motion for each time point to remove signal changes that were correlated with head/brain motion). Differences between trial types were estimated by contrasting the average peak response within the window of $4-8 \mathrm{sec}$ after stimulus onset (to account for hemodynamic delay). Differences between trial types were estimated for each participant individually and then combined in a second-stage random-effects analysis that identified differences in evoked responses that were consistent across participants. Regions exhibiting a significant effect by this random-effects analysis were those in which each voxel exhibited a reliable change in activity across participants $\left(t_{(11)}>3.5 ; p<0.005\right.$ uncorrected) in a $500 \mathrm{~mm}^{3}$ region (equal to 32 voxels in the $2.5 \mathrm{~mm}^{3}$ resolution of normalized space or $\sim 6$ voxels in the original anatomical space). Monte Carlo simulations using normally distributed noise with 750 time points (equivalent to the five runs) and 12 simulated participants indicate $<5 \%$ false positives per experiment with this statistical threshold.

\section{RESULTS}

Recognition memory performance on the post-scanning test demonstrated the expected directed forgetting effects for words, as shown in Figure 1. Participants endorsed more words that had been given with the cue to remember (R-words) than words given with the cue to forget (F-words) $\left(t_{(11)}=4.53 ; p<0.001\right)$. In addition, participants exhibited successful memory for both Rwords and F-words by endorsing these at a higher rate than novel words $\left(t_{(11)}>6.48 ; p<0.001\right)$. For face stimuli, participants exhibited successful memory for the faces seen during scanning $\left(t_{(11)}>4.56 ; p<0.001\right)$ but did not exhibit a directed forgetting effect, because the endorsement rates for R-faces and F-faces were not reliably different $\left(t_{(11)}=1.49 ; p>0.10\right)$.

\section{Words}

The robust effect of the remember/forget cue on encoding words was reflected in a network of brain regions that exhibited increased activity for R-words compared with F-words (Fig. 2, Table 1). Regions that exhibited increased activity during remember trials included a sizeable portion of LIPFC. This region includes both the anterior-ventral and posterior-dorsal areas observed in many previous verbal encoding studies (Wagner et al., 1998; Kirchhoff et al., 2000; Davachi et al., 2001; Otten et al., 2001). In addition, increased activity was observed in the anterior cingulate, middle superior frontal gyrus, left middle temporal gyrus, right insula, and right cerebellum.

Responses on the subsequent recognition test were used to sort study trials into those subsequently remembered (i.e., participants correctly responded "old") and those subsequently forgotten (i.e., participants incorrectly responded "new"). Activity thus associated with successful encoding was contrasted regardless of the remember/forget cue. Brain regions that exhibited increased activity for subsequently remembered words (Fig. 3, Table 2) in- cluded left parahippocampal cortex and posterior hippocampus, LIPFC, right superior parietal cortex, and right cerebellum.

The previous two contrasts reinforce the roles of both prefrontal cortex and left medial temporal lobe in episodic encoding. In addition, because the cue associated with each word was not completely predictive of subsequent memory (i.e., some R-words were forgotten and some F-words were remembered), it was possible to compare the effect of the cue and subsequent memory performance separately. In this analysis, each word was classified by both the subsequent memory outcome [successful (SM); unsuccessful (UM)] and the cue [remember (R); forget (F)]. The four possible conditions were thus SM-R, SM-F, UM-R, and UM-F. There were an average of 41.2 SM-R trials for each participant (range, 19-55), an average of 24.6 SM-F trials (range, 9-43), an average of 31.2 UM-R trials (range, 17-55), and an average of 47.9 UM-F trials (range, 25-66).

The critical comparison is between R-words that were not successfully remembered (UM-R) and F-words that were successfully remembered despite the cue to forget them (SM-F). To identify areas in which activity was more associated with the remember/forget cue than with subsequent memory, differential activity associated with UM-R and SM-F trials was compared within the network of brain areas already associated with the remember cue. [Note that this comparison is effectively the difference between the cue effect and the subsequent memory effect. The effect of the cue $=(\mathrm{SM}-\mathrm{R}+\mathrm{UM}-\mathrm{R})-(\mathrm{SM}-\mathrm{F}+\mathrm{UM}-\mathrm{F})$ and the subsequent memory effect $=(\mathrm{SM}-\mathrm{R}+\mathrm{SM}-\mathrm{F})-(\mathrm{UM}-\mathrm{R}+$ UM-F). Contrasting these two subtractions (cue $-\mathrm{SM}$ ) reduces to $2 * \mathrm{UM}-\mathrm{R}-2 * \mathrm{SM}-\mathrm{F}$, a linear combination of (statistically equivalent to) the UM-R - SM-F contrast used.] Restricting the analysis to just these regions (identified previously with a fairly stringent statistical threshold) reduced the number of concurrent comparisons. Accordingly, we identified clusters of voxels with consistently higher activity for UM-R trials than for SM-F trials at a threshold of $\mathrm{t}_{(11)}>2.20$ ( $p<0.05$ uncorrected). By this analysis, three areas were identified: anterior cingulate, $x=-6$, $y=+21, z=+33$ (volume $=1031 \mathrm{~mm}^{3}$, comprising $98.5 \%$ of the region within which it was located); superior frontal gyrus, $x=$ $-10, y=0, z=+60$ (volume $=984 \mathrm{~mm}^{3}, 97 \%$ of the region in which it was located); and anterior left inferior prefrontal cortex, $x=-50, y=+28, z=+6$ (volume $=797 \mathrm{~mm}^{3}, 11 \%$ of the region in which it was located). The identified areas were of sizeable spatial extent (797-1031 $\left.\mathrm{mm}^{3}\right)$ and encompass a large percentage of the source region of interest (ROI) for the superior frontal gyrus and anterior cingulated, making it highly unlikely that the differences were observed by chance. 

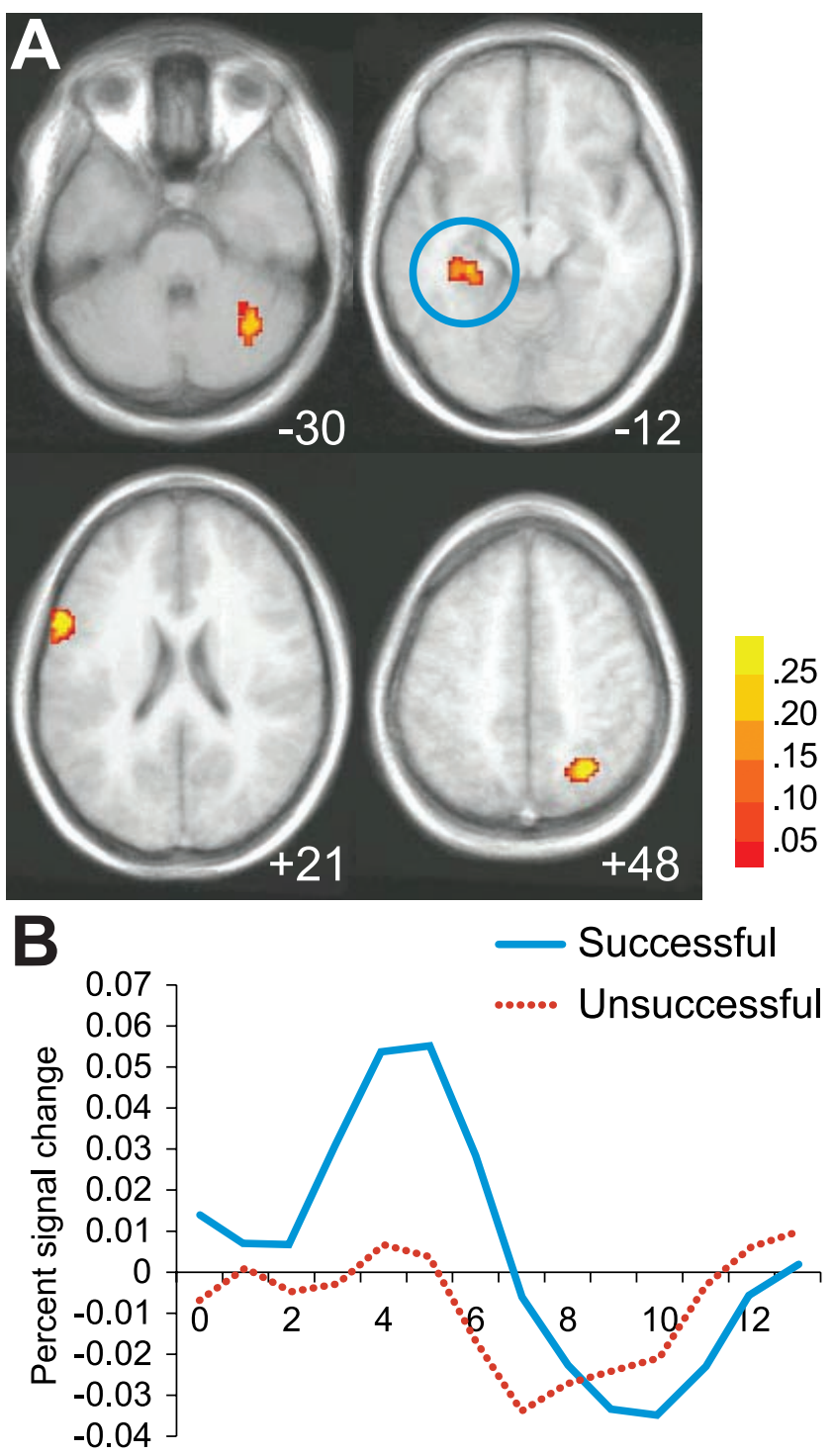

Time (s)

Figure 3. A, Brain areas in which increased activity was observed during word stimuli that were subsequently remembered on the recognition test compared with word stimuli that were not. Regions showing a reliable increase in activity across the group are shown in color overlaid on axial slices from the averaged high-resolution structural images. Regions shown are those for which the difference in peak activity between remembered and forgotten was consistently greater than zero across the group of participants $\left(t_{(11)}>3.5\right.$ in a cluster $>500 \mathrm{~mm}^{3}$ in volume). Color intensity (red/orange/yellow) indicates the magnitude of the average signal change between the two conditions. The level of the images within the standard atlas is noted. $B$, Time course of observed activity in the left posterior hippocampus and parahippocampal cortex region indicated with the blue circle on $A$ for successful and unsuccessful memory trials. The time course shown reflects averaged estimates of activity for each time point across all voxels in the region for all participants (with slight temporal smoothing for display purposes).

A similar analysis examined regions that exhibited increased activity for encoding success rather than encoding cue. In other words, activations for SM-F trials versus UM-R trials indicated which areas were reliably associated with successful encoding as opposed to the attempt to encode. Two such subregions were identified: left parahippocampal cortex, $x=-28, y=-35, z=$ -12 (volume $=391 \mathrm{~mm}^{3}, 70 \%$ of the region in which it was located); and right superior parietal cortex, $x=+32, y=-62, z=$ +45 (volume $=156 \mathrm{~mm}^{3}, 14 \%$ of the region in which it was located). This left medial temporal region and associated fMRI responses are shown in Figure 4, $C$ and $D$.

To further verify that the analysis comparing the SM-F and UM-R trials effectively captures differential activity for the regions examined, an ROI analysis was done by combining the data for all voxels within each region that exhibited increased activity for the remember cue and then comparing the peak activity for the UM-R and SM-F trial types estimated from the resulting time series. This analysis showed that the anterior cingulate and superior frontal gyrus regions each exhibited consistently higher activity for the UM-R trials $\left(t_{(11)}>3.25 ; p<0.01\right)$. The regionwide effect in the LIPFC was only marginal $\left(t_{(11)}=1.83 ; p<\right.$ $0.10)$, reflecting the fact that the region exhibiting increased UM-R activity was only a portion of the entire ROI. It is worth noting that this analysis is strongly affected by a single outlying participant who performed much worse than the rest of the group on the memory post-test (remembering $25 \%$ of the R-words, compared with the group average of 57\%), suggesting that this participant may have failed to use an effective encoding strategy. Adjusting the analysis to weight functional activity by memory performance or holding aside this outlier finds the effect to be reliable across the rest of the group $\left(t_{(11)}=2.21, p<0.05\right.$ and $t_{(10)}=2.59, p<0.05$, respectively). Another possible reason for the weaker ROI-wide response in the LIPFC is that the large LIPFC area that exhibited greater activity for "remember" than "forget" trials may include multiple sub-areas (Kirchhoff et al., 2000). Within this ROI, only an anterior, ventral subregion (Fig. $4 A, B)$ exhibited reliably more activity for the cue to remember compared with successful memory (in the first voxel-based analysis above). The posterior portion of this ROI exhibited increased activity for successful memory as well as for the remember cue.

A similar analysis was performed for the combined voxels within each ROI that exhibited increased activity for successful memory. Reliably increased activity was observed for the SM-F trials compared with UM-R trials in both the left parahippocampal region and the right superior parietal region $\left(t_{(11)}=3.48, p<\right.$ 0.01 and $t_{(11)}=2.51, p<0.03$, respectively).

\section{Faces}

Because participants did not exhibit a robust directed forgetting effect for faces, it is not surprising that few brain areas showed differential activity for faces associated with the remember cue (R-faces) versus the forget cue (F-faces). Regions with greater activity for R-faces and F-faces are shown in Figure 5; coordinates are listed in Table 3. For R-faces, increased activity was observed in left posterior occipital cortex, left fusiform gyrus, and right inferior occipitotemporal cortex. In addition, two areas exhibited greater activity for F-faces: right parietal cortex and right middle frontal gyrus. It is unclear whether these areas reflect activity associated with an attempt to forget or some other cognitive activity that occurs after the forget cue (e.g., rehearsal of other previously presented stimuli, vigilance for the next stimulus).

No brain areas showed greater encoding activity for successfully remembered faces compared with forgotten faces. This absence of subsequent memory effects is likely attributable to the low recognition accuracy for faces. Given the high false alarm rate (30\% of the novel faces were incorrectly endorsed as old) relative to the hit rate $(40 \%$ of the old faces were correctly endorsed as 
Table 2. Regions that exhibited greater activity for subsequently remembered words than for subsequently forgotten words

\begin{tabular}{llrrr} 
Brain region & $\begin{array}{l}\text { Brodmann's } \\
\text { area (BA) }\end{array}$ & $x$ & $y$ & $\begin{array}{c}\text { Volume } \\
\left(\mathrm{mm}^{3}\right)\end{array}$ \\
\hline Right superior parietal cortex & BA 7 & 29 & -61 & 46 \\
Left inferior prefrontal cortex & BA 45 & -58 & 10 & 21 \\
Right cerebellum & & 34 & -59 & -30 \\
Left parahippocampal cortex and posterior hippocampus & BA 36 & -30 & -34 & -12 \\
\hline
\end{tabular}

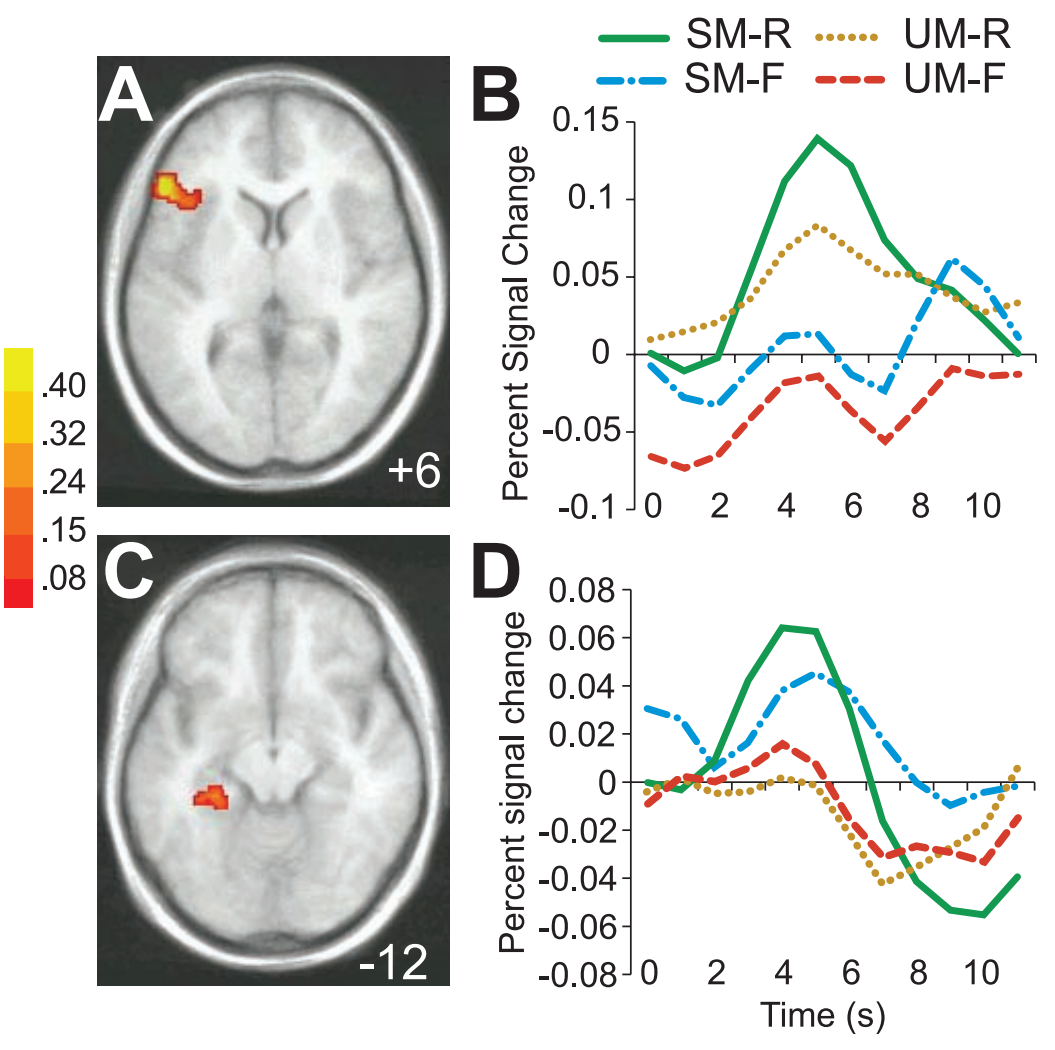

Figure 4. Comparison of encoding effort and success in the left inferior prefrontal cortex and medial temporal lobe. $A$, The LIPFC region in which encoding effort was more predictive of activity than encoding success (for words). $B$, Time courses of observed activity for the four trial types: successfully remembered R-words $(S M-R)$, unsuccessfully remembered R-words $(U M-R)$, successfully remembered F-words $(S M-F)$, and unsuccessfully remembered F-words $(U M-F)$. Of particular note is the fact that this area exhibits a strong response to R-words, even when this effort does not produce successful memory (UM-R $>$ SM-F). $C$, The left MTL region in which encoding success was more predictive of activity than encoding effort (for words). $D$, Time courses of observed activity in the MTL region for the four trial types. In this region, successful encoding is associated with an increase in activity, even after the forget cue, whereas an unsuccessful encoding attempt is not associated with increased activity (SM-F > UM-R). old), a large proportion of faces considered to be subsequently remembered may reflect guessing rather than a veridical memory.

Brain areas in which activity differed for word and face trials are listed in Table 4. Areas where words evoked consistent activity included the LIPFC (Broca's area) and left superior temporal gyrus (Wernicke's area). Face stimuli evoked more activity in the right amygdala and parahippocampal gyrus.

\section{DISCUSSION}

\section{Encoding words}

The conjoint analysis of subsequent memory effects and directed forgetting effects yielded evidence that left inferior prefrontal cortex and the medial temporal lobe play different roles in verbal encoding. Based on the directed forgetting procedure, increased encoding effort on remember trials was associated with increased activity in LIPFC. Furthermore, comparisons between successful and unsuccessful encoding based on subsequent recognition revealed what have been termed declarative memory (DM) effects (Paller and Wagner, 2002). Increased left MTL activity was thus associated with the successful formation of declarative memories.

Although effort and success were correlated, high effort did not always lead to success and low effort did not always lead to forgetting. Therefore, it was possible to separate these factors by comparing trials when recognition was unsuccessful despite a remember cue (UM-R trials) and trials when successful recognition followed a forget cue (SM-F trials). The anterior, ventral portion of LIPFC exhibited greater activity for UM-R trials, indicating that activity in this brain area was more closely associated with the effort to encode a new memory than with successful creation of a new memory. In contrast, left MTL exhibited greater activity for SM-F trials, indicating that activity in that area was more closely linked with the creation of a memory that could be retrieved subsequently than with the intention to create a memory.

These two areas, LIPFC and left MTL, have been found in several previous studies to coactivate as part of a circuit associated with successful memory encoding (Wagner et al., 1998; Kirchhoff et al., 2000; Baker et al., 2001; Otten and Rugg, 2001; Otten et al., 2001; Strange et al., 2002). Factors that increase the effectiveness of encoding, such as instructions to remember or deeper processing in a levels-of-processing manipulation, might be expected to increase activity throughout this circuit. In the current study, both areas were strongly activated for successfully remembered trials that included the remember cue (SM-R trials). In earlier investigations, comparisons between successful and unsuccessful encoding were based on variability in memory re- 


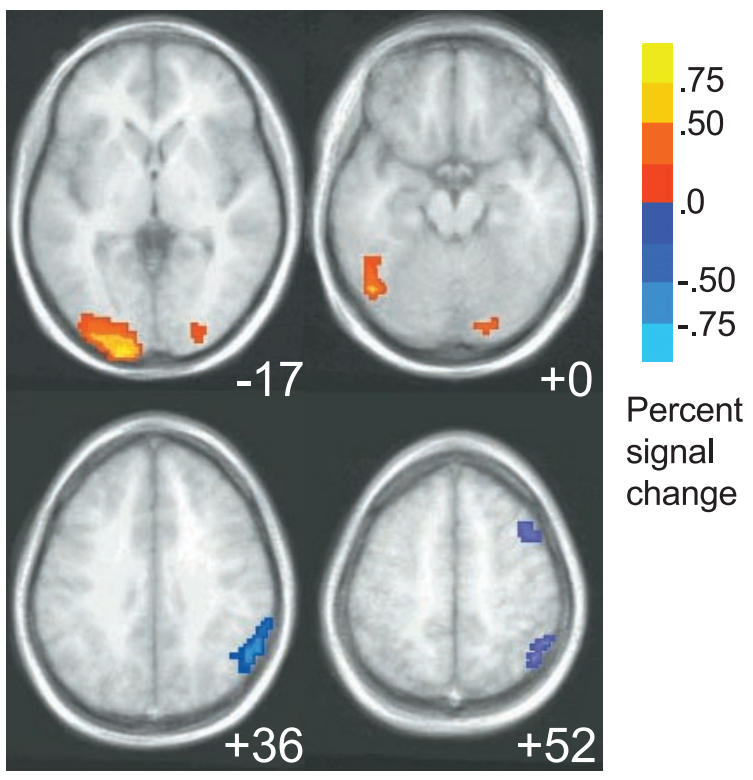

Figure 5. Brain areas in which increased activity was observed during face stimuli cued to be remembered (R-faces) compared with face stimuli cued to be forgotten (F-faces). Regions showing a reliable increase in activity across the group are shown in color overlaid on axial slices from the averaged high-resolution structural images. Regions shown are those for which the difference in peak activity between R-faces and F-faces was consistently different from zero across the group of participants $\left(t_{(11)}>\right.$ 3.5 in a cluster $>500 \mathrm{~mm}^{3}$ in volume). Warm colors (red/orange/yellow) indicate areas in which increased activity was observed for R-faces. Cool colors (blue/cyan) indicate areas in which increased activity was observed during F-face trials. The level of the images within the standard atlas is noted.

sulting from stimulus differences, varying levels of attention and effort put forth across stimuli, and other uncontrolled factors. By including cues to remember or forget in the present experiment and presenting the cue after each stimulus presentation, we reduced the effects of fluctuating attention from trial to trial on the mnemonic fate of each stimulus. Because this directed forgetting procedure manipulated encoding effort and effectiveness in a direct manner, we were able to disentangle the roles of LIPFC and MTL in encoding new episodic memories.

Although our a priori hypotheses concerned the roles of the LIPFC and MTL in episodic encoding, similar patterns of activity were also observed in other areas. The anterior cingulate and medial superior frontal gyrus both exhibited patterns of activity similar to LIPFC. These findings suggest that these additional frontal areas may act in concert with LIPFC as part of a network supporting verbal encoding effort. Right parietal cortex exhibited a pattern of activity similar to that of left MTL, suggesting that this area may be acting to support successful encoding of an episode. This right parietal area was also identified by Davachi et al. (2001) as exhibiting increased activity during rote word rehearsal that led to successful memory. Processing in this region may play a supportive or subsidiary role in encoding, given the ample neuropsychological evidence that the MTL is critical for memory (Scoville and Milner, 1957; Squire, 1992). The parietal area may process details of the spatiotemporal context of the episode, given that the context of the encoding episode is necessary to later remember the episode and recognize the preexperimentally familiar words on the test.

Two additional regions were identified as active during both encoding effort and success. The posterior dorsal part of the
LIPFC encoding area and the right cerebellum both exhibited increased activity in response to the remember cue and when encoding was successful, without preferentially activating in either contrast. This pattern of results suggests that these two areas are active when encoding is attempted and when it is successful. However, it is unclear whether these areas are engaged in a cognitive process common to both of these conditions or whether these areas play different cognitive roles depending on context. One possibility for a common process would be linking a to-beremembered word to semantic memory in an elaborative manner (an effortful and successful encoding strategy).

Although inferences from neuropsychology did not originally point to PFC as a necessary component of episodic encoding networks, neuroimaging results have repeatedly implicated PFC. Manipulations that increase encoding effectiveness, such as deep or elaborative encoding, consistently produce increased activity in left prefrontal cortex (Kapur et al., 1994, 1996; Buckner et al., 1999). These manipulations may increase the effort that participants devote to encoding or may reflect common mechanisms engaged when encoding effectiveness is increased by any factor that improves subsequent memory. Although we did not provide participants with instructions to use specific encoding strategies during remember-cued trials, we expect that they engaged in various elaborative encoding strategies, rote rehearsal, and other mnemonic devices. The superior recognition of words that were cued to be remembered indicates that these strategies were mostly successful. Although LIPFC activity was observed on UM-R trials (when encoding effort was unsuccessful), LIPFC may be part of a network specifically engaged for verbal memory processing, perhaps for both rote rehearsal and semantic elaboration. A related hypothesis is that LIPFC may play a supporting or modulating role in episodic encoding through excitatory inputs to MTL. LIPFC may help to precipitate processing in MTL that functions to link various verbal and contextual features together, so as to facilitate later retrieval of the encoding episode.

\section{Encoding faces}

In contrast to the effectiveness of DF cues on encoding of words, the same cues had minimal effects on encoding novel faces. Given the absence of a significant behavioral difference, it is not surprising that little differential activity was observed related to the cue. Despite this, increased activity was observed for remember trials in three posterior cortical areas. Because the cue followed the faces, this activity may reflect efforts to visualize the face after the remember cue. In right midfrontal and parietal cortex, activity increased during forget trials. This may reflect active attempts to forget or to rehearse to-be-remembered stimuli from previous trials during a forget trial. If displaced rehearsal took place, it would remove power from both DF and subsequent memory analyses.

During the subsequent recognition test, participants produced a high false alarm rate to novel faces, complicating the subsequent memory analysis for faces by calling into question the proportion of correct old responses that reflected veridical memories rather than correct guessing. The resulting decrease in power likely caused the lack of subsequent memory effects observed for faces. Because of this limitation in DM studies, future test conditions should influence response criteria to minimize the false alarm rate. It is also critical that the difference between the correct old (hit) rate and the miss rate is not too large; at maximal recognition performance, power is greatly reduced for subsequent memory analysis because of a low number of forgotten trials. 
Table 3. Regions that exhibited different levels of activity for remember and forget trials for face stimuli

\begin{tabular}{|c|c|c|c|c|c|}
\hline Brain region & $\begin{array}{l}\text { Brodmann's } \\
\text { area (BA) }\end{array}$ & $x$ & $y$ & $z$ & $\begin{array}{l}\text { Volume } \\
\left(\mathrm{mm}^{3}\right)\end{array}$ \\
\hline \multicolumn{6}{|l|}{ Remember $>$ forget } \\
\hline Left middle and inferior occipital gyrus & BA $17 / 18$ & -22 & -96 & 1 & 7500 \\
\hline Left fusiform gyrus & BA 37 & -46 & -64 & -17 & 1109 \\
\hline Right inferior occipital/posterior fusiform gyrus & BA 18 & 23 & -89 & -10 & 625 \\
\hline \multicolumn{6}{|l|}{ Forget $>$ remember } \\
\hline Right parietal cortex & BA $39 / 40$ & 54 & -55 & 37 & 4938 \\
\hline Right middle frontal gyrus & BA 6 & 42 & 12 & 52 & 625 \\
\hline
\end{tabular}

Table 4. Regions that exhibited differential activity for face and word stimuli

\begin{tabular}{|c|c|c|c|c|c|}
\hline Brain region & $\begin{array}{l}\text { Brodmann's } \\
\text { area (BA) }\end{array}$ & $x$ & $y$ & $z$ & $\begin{array}{l}\text { Volume } \\
\left(\mathrm{mm}^{3}\right)\end{array}$ \\
\hline \multicolumn{6}{|l|}{ Word $>$ face } \\
\hline Left prefrontal cortex and insula & BA 9, 45, 46, 47 & -49 & 14 & 18 & 18828 \\
\hline Left superior temporal gyrus & BA 22 & -60 & -42 & 6 & 6375 \\
\hline Medial frontal gyrus & BA 6 & -12 & 5 & 54 & 5297 \\
\hline Right superior parietal cortex & BA 7 & 36 & -59 & 49 & 4656 \\
\hline Right middle frontal gyrus & BA 6 & 27 & 6 & 53 & 4562 \\
\hline Left superior parietal cortex & BA $7 / 39$ & -33 & -62 & 43 & 3594 \\
\hline Right superior frontal gyrus & BA $9 / 10$ & 35 & 43 & 26 & 3266 \\
\hline Left middle occipital gyrus & BA 18 & -20 & -97 & 1 & 2203 \\
\hline Right cerebellum & & 25 & -68 & -32 & 1844 \\
\hline Medial parietal cortex/precuneus & BA 7 & -10 & -65 & 55 & 1266 \\
\hline Postcentral gyrus & BA 3 & 26 & -28 & 70 & 906 \\
\hline Left insula & BA 13 & -32 & -20 & -1 & 500 \\
\hline \multicolumn{6}{|l|}{ Face $>$ word } \\
\hline Right parahippocampal gyrus & BA 37 & 28 & -48 & -6 & 688 \\
\hline Right amygdala & & 18 & -8 & -13 & 547 \\
\hline
\end{tabular}

Stimulus-specific effects were found for words and faces (Table 4). Increased activity for words was found throughout areas that have classically shown greater involvement in language processing. Increased activity for faces was observed only in the right amygdala and parahippocampal gyrus. Although the fusiform gyrus was not active in the group analysis, many individual subjects exhibited increases in this area [reflecting the fusiform face area of Kanwisher et al. (1997)]. The general lack of activity for face stimuli suggests that there may have been some perceptual difficulty associated with the faces, possibly caused by poor image contrast. The greater stimulus-correlated activity for words reinforces the fact that for our study, the responses to word stimuli are more informative about the PFC and MTL circuitry that supports memory encoding.

\section{Conclusion}

By manipulating encoding through post-stimulus directed forgetting instructions, we identified an association between the intention to encode the episode of viewing a word and increased activity in left inferior prefrontal cortex. By examining neural activity that was predictive of subsequent success in remembering across the same trials, we identified a different association between successful verbal encoding and left medial temporal activation. We conclude that these two areas collaborate in a circuit that supports the encoding of episodic memories for verbal materials, but that these two regions play divergent roles. In the normal course of operation, the intention to encode results in increased activity in left inferior prefrontal cortex. This activity in turn can potentially modulate activity in the medial temporal lobe, where an increase in activity may reflect the storage of the multidimensional links that effectively support successful encoding.

\section{REFERENCES}

Baker JT, Sanders AL, Maccotta L, Buckner RL (2001) Neural correlates of verbal memory encoding during semantic and structural processing tasks. NeuroReport 12:1251-1256.

Bjork RA (1989) Retrieval inhibition as an adaptive mechanism in human memory. In: Varieties of memory and consciousness, pp 309-330. Hillsdale, NJ: Erlbaum.

Brewer JB, Zhao Z, Desmond JE, Glover GH, Gabrieli JDE (1998) Making memories: brain activity that predicts how well visual experience will be remembered. Science 281:1185-1187.

Buckner RL, Kelley WM, Petersen SE (1999) Frontal cortex contributes to human memory formation. Nat Neurosci 2:311-314.

Collins DL, Neelin P, Peters TM, Evans AC (1994) Automatic 3D intersubject registration of MR volumetric data in standardized Talairach space. J Comput Assist Tomogr 18:192-205.

Cox RW (1996) AFNI: Software for analysis and visualization of functional magnetic resonance neuroimages. Comput Biomed Res 29:162-173.

Davachi L, Maril A, Wagner AD (2001) When keeping in mind supports later bringing to mind: neural markers of phonological rehearsal predict subsequent remembering. J Cognit Neurosci 13:1059-1070.

Golding JM, MacLeod CM (1998) Intentional forgetting: interdisciplinary approaches. Mahwah, NJ: Erlbaum.

Kanwisher N, McDermott J, Chun MM (1997) The fusiform face area: a module in human extrastriate cortex specialized for face perception. J Neurosci 17:4302-4311.

Kapur S, Craik FIM, Tulving E, Wilson AA, Houle S, Brown GM (1994) Neuroanatomical correlates of encoding in episodic memory: levels of processed effect. Proc Natl Acad Sci USA 91:2008-2011. 
Kapur S, Tulving E, Cabeza R, McIntosh AR, Houle S, Craik FIM (1996) The neural correlates of intentional learning of verbal materials: a PET study in humans. Cognit Brain Res 4:243-249.

Kirchhoff BA, Wagner AD, Maril A, Stern CE (2000) Prefrontaltemporal circuitry for episodic encoding and subsequent memory. J Neurosci 20:6173-6180.

Kucera H, Francis WN (1967) Computational analysis of present-day American English. Providence: RI: Brown UP.

Otten LJ, Rugg MD (2001) Task-dependency of the neural correlates of episodic encoding as measured by fMRI. Cereb Cortex 11:1150-1160.

Otten LJ, Henson RNA, Rugg MD (2001) Depth of processing effect on neural correlates of memory encoding: relationship between findings from across- and within-task comparison. Brain 124:399-412.

Paller KA, Wagner AD (2002) Observing the transformation of experience into memory. Trends Cognit Neurosci 6:93-102.

Scoville WB, Milner B (1957) Loss of recent memory after bilateral hippocampal lesions. J Neurol Neurosurg Psychiatr 20:11-21.
Squire LR (1992) Memory and the hippocampus: a synthesis from findings with rats, monkeys, and humans. Psychol Rev 99:195-231.

Squire LR, Knowlton BJ (2000) The medial temporal lobe, the hippocampus, and the memory systems of the brain. In: The new cognitive neurosciences, Ed 2 (Gazzaniga MS, ed), pp 765-779. Cambridge, MA: MIT.

Squire LR, Schacter DL (2002) The neuropsychology of memory, Ed 3. New York: Guilford.

Strange BA, Otten LJ, Josephs O, Rugg MD, Dolan RJ (2002) Dissociable human perirhinal, hippocampal, and parahippocampal roles during verbal encoding. J Neurosci 22:523-528.

Talairach J, Tournoux P (1988) A coplanar stereotaxic atlas of the human brain. Stuttgart: Thieme.

Wagner AD, Schacter DL, Rotte M, Koutstaal W, Maril A, Dale AM, Rosen BR, Buckner RL (1998) Building memories: remembering and forgetting of verbal experiences as predicted by brain activity. Science 281:1188-1191. 